\title{
Production of Sn and Sb isotopes in high-energy neutron-induced fission of ${ }^{\text {nat }} U$
}

\author{
A. Mattera ${ }^{1, a}$, S. Pomp ${ }^{1, b}$, M. Lantz ${ }^{1}$, V. Rakopoulos ${ }^{1}$, A. Solders ${ }^{1}$, A. Al-Adili ${ }^{1}$, H. Penttilä ${ }^{2}$, I.D. Moore ${ }^{2}$, \\ S. Rinta-Antila ${ }^{2}$, T. Eronen ${ }^{2}$, A. Kankainen ${ }^{2}$, I. Pohjalainen ${ }^{2}$, D. Gorelov ${ }^{2}$, L. Canete ${ }^{2}$, D. Nesterenko ${ }^{2}$, M. Vilén ${ }^{2}$, \\ and J. Äystö ${ }^{2}$ \\ 1 Uppsala University, BOX 516, 75120 Uppsala, Sweden \\ 2 University of Jyväskylä, P.O. Box 35 (YFL), 40014 Jyväskylä, Finland
}

Received: 15 August 2017 / Revised: 19 December 2017

Published online: 5 March 2018

(c) The Author(s) 2018. This article is published with open access at Springerlink.com

Communicated by N. Kalantar-Nayestanaki

\begin{abstract}
The first systematic measurement of neutron-induced fission yields has been performed at the upgraded IGISOL-4 facility at the University of Jyväskylä, Finland. The fission products from high-energy neutron-induced fission of ${ }^{n a t} U$ were stopped in a gas cell filled with helium buffer gas, and were online separated with a dipole magnet. The isobars, with masses in the range $A=128-133$, were transported to a tape-implantation station and identified using $\gamma$-spectroscopy. We report here the relative cumulative isotopic yields of tin $(Z=50)$ and the relative independent isotopic yields of antimony $(Z=51)$. Isomeric yield ratios were also obtained for five nuclides. The yields of tin show a staggered behaviour around $A=131$, not observed in the ENDF/B-VII.1 evaluation. The yields of antimony also contradict the trend from the evaluation, but are in agreement with a calculation performed using the GEF model that shows the yield increasing with mass in the range $A=128-133$.
\end{abstract}

\section{Introduction}

Fission Yields (FYs) are one of the main quantities of interest to understand the fission process. FY distributions offer an insight into the characteristics at scission of the compound nucleus undergoing fission. More and better fission yield data for various fissioning systems and incoming neutron energies are needed for Gen-IV reactors and accelerator-driven systems. Nevertheless, except for a handful of better-studied fissioning systems and energies (such as ${ }^{235} \mathrm{U}\left(\mathrm{n}_{\text {therm. }}, \mathrm{f}\right),{ }^{239} \mathrm{Pu}\left(\mathrm{n}_{\text {therm. }}, \mathrm{f}\right),{ }^{252} \mathrm{Cf}(\mathrm{SF})$ ), data are scarce and have in many cases large uncertainties [1]. A recent technical report from the IAEA also highlighted the need for new high-quality fission yield measurements [2].

In this work we report on the first successful measurement of mass-separated products from fission of ${ }^{\text {nat }} U$ induced by high-energy neutrons performed at the upgraded IGISOL-4 facility in JYFL-ACCLAB, University of Jyväskylä [3]. Relative isotopic yields were extracted for tin (Sn) in the mass range $A=128-132$ and for antimony (Sb) in the mass range $A=128-133$. In this region, a recent experiment by Wilson et al., on fission of ${ }^{238} \mathrm{U}$

\footnotetext{
a e-mail: andrea.mattera@gmail.com

b e-mail: stephan.pomp@physics.uu.se
}

just above the fission threshold, found large discrepancies between the measured yields and the ones from the JEFF 3.1.1 and GEF models [4].

\section{Experimental method}

The Ion Guide Isotope Separator On-Line (IGISOL) facility, at JYFL, has been used in the past for measurements of proton-induced fission yields using the IGISOL technique, often coupled to the JYFLTRAP Penning trap for the identification and counting of the fission products $[5,6]$.

In this experiment, the high-energy neutrons were produced by $30 \mathrm{MeV}$ protons from the K130 cyclotron impinging on a $6 \mathrm{~mm}$-thick beryllium converter target, similar to the one described in refs. $[6,7]$. The neutron energy distribution is that of a white neutron spectrum extending to about $30 \mathrm{MeV}$. The neutron field was used to induce fission in two $(1 \times 5) \mathrm{cm}^{2}$ and $15 \mathrm{mg} / \mathrm{cm}^{2}$ thick ${ }^{\text {nat }} \mathrm{U}$ foils. The two foils were attached to a thin aluminium support and installed in a specifically designed ion guide [8] at a distance of $2.5 \mathrm{~cm}$ from the back of the neutron source.

The average neutron energy weighted with the fission cross section of ${ }^{238} \mathrm{U}$, that accounts for $(98 \pm 1) \%$ of the fission events, is $(12.4 \pm 8.8) \mathrm{MeV}$. We also estimated the 
Table 1. IYRs measured in this experiment, compared to the ENDF/B-VII.1 evaluated data library for fission of ${ }^{238} \mathrm{U}$ induced by $14 \mathrm{MeV}$ neutrons, and to a GEF calculation with a realistic incoming neutron spectrum. IYRs are reported as $Y_{i s} /\left(Y_{i s}+Y_{G S}\right)$. The values reported are cumulative IYRs and independent IYRs for Sn and Sb, respectively.

\begin{tabular}{ccccccc}
\hline & $J_{i s}^{\Pi}$ & $J_{G S}^{\Pi}$ & $E_{i s}^{*}(\mathrm{keV})$ & This work & GEF & ENDF B-VII.1 \\
\hline${ }^{129} \mathrm{Sn}$ & $(11 / 2)^{-}$ & $(3 / 2)^{+}$ & $35.15(5)$ & $0.37(13)$ & 0.63 & $0.15(12)$ \\
${ }^{130} \mathrm{Sn}$ & $7^{-}$ & $0^{+}$ & $1946.88(10)$ & $0.64(48)$ & 0.49 & $0.48(49)$ \\
${ }^{131} \mathrm{Sn}$ & $(11 / 2)^{-}$ & $(3 / 2)^{+}$ & $65.1(3)$ & $0.43(19)$ & 0.69 & $0.48(45)$ \\
\hline${ }^{130} \mathrm{Sb}$ & $(4,5)^{+}$ & $8^{-}$ & $4.80(20)$ & $0.81(43)$ & 0.68 & $0.43(41)$ \\
${ }^{132} \mathrm{Sb}$ & $8^{-}$ & $4^{+}$ & $200(30)$ & $0.25(10)$ & 0.49 & $0.61(41)$ \\
\hline
\end{tabular}

probability of multi-chance fission for ${ }^{238} \mathrm{U}$ using the GEF code [9], that resulted in $55 \%, 31 \%, 8 \%$ and $6 \%$ for first, second, third and fourth chance fission, respectively. Additional details on the neutron energy spectrum can be found in refs. [7,10].

The fission fragments were stopped in helium gas at a pressure of 400 mbar (with about $4 \%$ stopping efficiency [11]) and extracted from the ion guide gas cell using a SextuPole Ion Guide (SPIG) [12] and differential pumping. The ions were then accelerated to $24 \mathrm{keV}$ and separated online based on their charge-over-mass ratio using a dipole magnet with a mass resolving power $M / \Delta M$ of about 500 .

The proton-to-neutron conversion ratio was estimated to be approximately $3.02 \times 10^{-3}$ neutrons/proton. Due to the low maximum current available for this experiment $(10 \mu \mathrm{A})$, and the resulting low fission rate, we chose not to transport the fission products to the Penning trap. The ions were instead collected on a movable implantation tape and identified using $\gamma$-spectroscopy.

The $\gamma$-spectroscopy measurement was performed with a Canberra GC7020 70\% coaxial p-type HPGe detector, with an energy resolution at $1332.5 \mathrm{keV}$ of $3.1 \mathrm{keV}$ (vs. a nominal resolution of $2 \mathrm{keV}$ ). The detector, efficiencycalibrated with an ${ }^{152} \mathrm{Eu}$ source, was operated in coincidence with a plastic scintillator that surrounded the implantation tape, used to detect the electrons from the $\beta^{-}$decay of the fission products. A $\gamma$-spectrum was acquired for each mass between $A=128$ and $A=133$. In order to reduce contamination from different mass chains, the implantation tape was moved after modifying the settings of the dipole magnet (i.e., after changing mass chain).

Data for each mass were measured for $7200 \mathrm{~s}$, except for mass $A=133$ that was measured for $3600 \mathrm{~s}$. Before acquisition, fission products were accumulated on the tape for times ranging from $60 \mathrm{~s}$ to $15 \mathrm{~min}$. This was done in order to get as close as possible to the saturation condition for the nuclides that dominate the respective $\gamma$-spectra.

The $\gamma$-spectra for all masses were analysed and the areas of the most prominent peaks were extracted using the fit and background-subtraction function of the FitzPeaks v3.2 code [13]. The determined intensities were corrected for the relative efficiency of the detector. When more than one peak was observed for a nuclide, the weighted average was used to obtain a value for the total number of decays. Peaks common to more than one nuclide (typi- cally, ground and isomeric states of a nuclide), were not used in the analysis. A list of all the gamma lines used in this analysis, their relative intensities, and the library from which the decay data were extracted, are tabulated in ref. [14].

If the precursors of the nuclide under analysis have reached the saturation condition when the measurement starts, the integrated activity $(A)$, over the measurement time $(T)$ can be extracted as

$$
A=\int_{T} P R \cdot\left(1-e^{-\lambda t}\right) \mathrm{d} t=P R \cdot\left[T-\frac{1-e^{-\lambda T}}{\lambda}\right],
$$

so that the production rate $(P R)$ - or Cumulative Fission Yields (CFY) - can be calculated as

$$
P R=\frac{A}{T-\frac{1-\exp (-\lambda T)}{\lambda}},
$$

where $\lambda$ is the decay constant of the studied nuclide.

This also allows the extraction of CFY for longer-lived nuclei, without any knowledge of the yield of the precursors, as long as these precursors have short half-lives. The assumptions needed to derive eq. (1) are satisfied for all isotopes of Sn and eq. (2) was used to extract the CFY.

In the analysis of Sb data, eq. (1) is not always valid, given the longer half-lives of the precursors (up to $59.1 \mathrm{~min}$ in the case of ${ }^{128} \mathrm{Sn}$, decaying to ${ }^{128 \mathrm{~m}} \mathrm{Sb}$ ). However, it was possible to use the CFY measured for Sn and to subtract the contribution of precursors to the yield of Sb, thus extracting Independent Fission Yields (IFY).

\section{Results}

Following the procedure outlined in sect. 2, the relative yields were obtained for five isotopes of Sn (128 to 132) and six isotopes of $\mathrm{Sb}$ (128 to 133). The yields are plotted in fig. 1. For three isotopes of $\mathrm{Sn}$ and two of $\mathrm{Sb}$ both ground and isomeric states were observed. In these cases, what is plotted is the sum of the two yields. Detailed results of the observed isomeric yield ratios (IYR) are presented in table 1.

The precise transport efficiency of the setup is not known experimentally. Based on previous experience and 


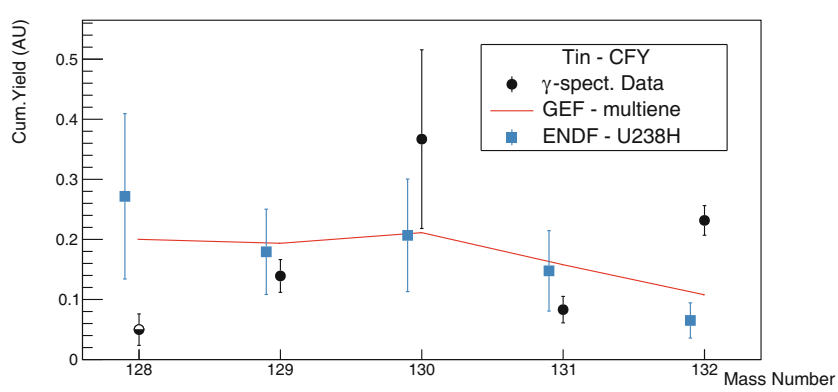

(a)

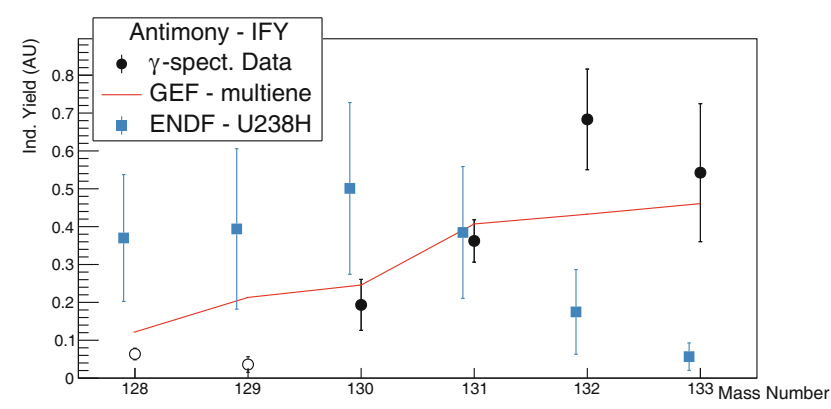

(b)

Fig. 1. Relative CFY of $\mathrm{Sn}$ (a) and relative IFY of $\mathrm{Sb}$ (b) produced in high-energy neutron-induced fission of ${ }^{\text {nat }} U$. The half-filled symbol $(\bullet)$ is used for ${ }^{128} \mathrm{Sn}$, where only the GS was seen. Open symbols (o) are used for ${ }^{128} \mathrm{Sb}$ and ${ }^{129} \mathrm{Sb}$, where only the isomeric states were observed due to the longer halflife of the GS.

on the simulation studies of ion stopping efficiency reported in refs. [11,15], we can safely assume that the efficiency does not change within the same atomic number, and does not change significantly for neighbouring masses. It was however not possible to refer the measured activities to the absolute number of fissions, hence only relative yields are presented in fig. 1, where the sum of the evaluated yields (ENDF [16] and GEF) was rescaled to the sum of the measured values.

\section{Discussion}

The fission products that reach the tape station are only a fraction of the number of ions produced in the fission reaction. Losses occur in recombination processes in the fission ion guide, as well as during the transport and the mass selection in the dipole magnet.

In fig. 1, the error bars on our experimental points show only the statistical uncertainty. The uncertainty on the initial proton current (estimated in about 5\%), as well as uncertainties due to decay data are not included.

The mechanisms of ion recombination of the highlyionized fission products down to charge $1^{+}$- the one generally selected by the dipole magnet - can depend on the chemistry of the produced elements and on the impurity concentration in the helium stopping gas. Thus, even though the IGISOL technique is able to produce all chemical elements and is considered element-independent [6], the total transport efficiency will ultimately depend on the $Z$ of the fission products.

We investigated in detail the ratio of singly- and doubly-charge ions at $A=132$. The results showed that the ratio $P R\left(1^{+}\right) / P R\left(2^{+}\right)$goes from $1.19 \pm 0.17$ for ${ }^{132} \mathrm{Sn}$ to $2.78 \pm 0.42$ for ${ }^{132} \mathrm{Sb}$. This suggests that comparing absolute values of the production rates of the two elements is not relevant, until a more complete understanding of the chemistry-dependent ion recombination in the ion guide is reached.

Nevertheless, it is necessary and justified to subtract the CFY for $\mathrm{Sn}$ in order to obtain IFY for $\mathrm{Sb}$, as discussed in sect. 2 . Since the half-lives of precursors are significantly longer than the transport time of FPs from production to implantation at the tape station, we can assume that the decays of $\mathrm{Sn}$ to $\mathrm{Sb}$ happen on the tape, i.e., after the element-dependent recombination and the $q / A$ selection by the dipole magnet. In this case, the CFY measured for Sn are the same that would contribute to the increased activity of $\mathrm{Sb}$ caused by $\beta$-decay of $\mathrm{Sn}$.

CFY of the Sn isotopes are shown in fig. 1(a). The precursors of $\mathrm{Sn}$ in all mass chains have a half-life shorter than $1.5 \mathrm{~s}$, allowing us to use the approximations discussed in sect. 2. The $7^{-}$isomer of ${ }^{128} \mathrm{Sn}$ decays through internal transition with a probability of $100 \%$ and it was not visible due to the $\beta$-coincidence condition used in this experiment.

In the case of $\mathrm{Sn}$, the cumulative yield exhibits a staggered behaviour around mass $A=131$, that is not observed in the ENDF/B-VII.1 evaluated data library for fission of ${ }^{238} \mathrm{U}$ induced by $14 \mathrm{MeV}$ neutrons. No indication of the even-odd staggering is either seen in a GEF calculation, that we made taking into account the full neutron energy spectrum obtained from a Monte Carlo simulation. This behaviour makes the results recently reported by Wilson et al. (ref. [4]) even more striking. In their work, normalization of the measured even-even yields is carried out by assuming no odd-even effects and this results in a yield 5-6 times lower than calculations done using the GEF and JEFF 3.1.1 models.

Decay information of ${ }^{131} \mathrm{Sn}$ was not available in ref. [16] and incomplete in ref. [17]. We used ref. [18] to discriminate between ${ }^{131} \mathrm{Sn}$ and ${ }^{131 \mathrm{~m}} \mathrm{Sn}$. This lack of information may have caused an incorrect estimation of the activity. In ref. [14], we provide all the necessary information to update the analysis, should new decay data become available.

The large uncertainty at $A=130$ is dominated by counting statistics. For ${ }^{130 \mathrm{~m}} \mathrm{Sn}\left(7^{-}\right)$, the only visible peak is at $898.5 \mathrm{keV}$, that - with an intensity of $8 \%$ - produces only $31 \pm 19$ counts in the peak in a measurement time of $7200 \mathrm{~s}$. The effect of this large uncertainty to ${ }^{130} \mathrm{Sb}$ IFY is limited since the Sn decay go predominantly to the ground state while fission populates mainly the metastable state (see table 1).

The relative IFYs measured for $\mathrm{Sb}$ are shown in fig. 1(b). The ground states of ${ }^{128} \mathrm{Sb}$ and ${ }^{129} \mathrm{Sb}$ have a 
half-life of $9.01 \mathrm{~h}$ and $4.4 \mathrm{~h}$, respectively, and were not observed in the $\gamma$-spectra. The behaviour observed shows an increasing trend from masses $A=128$ to 132 , that results in a peak shifted by as many as two mass units above the one seen in the ENDF/B-VII.1 library for $14 \mathrm{MeV}$ neutron-induced fission of ${ }^{238} \mathrm{U}$. Our measurement seems instead to confirm the calculations performed with the GEF code.

The uncertainties on the IYRs shown in table 1 are rather large, especially if compared to previous experimental campaigns at the same facility, that used direct ion counting with JYFLTRAP for nuclide identification [19]. In addition, the large uncertainties on the Evaluated $\mathrm{Nu}$ clear Data Libraries, also make it difficult to highlight any clear trend.

Nevertheless, it is possible to observe a certain consistency in the results from this measurement when comparing, e.g. ${ }^{129} \mathrm{Sn}$ and ${ }^{131} \mathrm{Sn}$ : these two isotopes carry the same spin assignment for the isomeric/ground state, and show the same IYR. We can also observe that the spin assignment for the two isotopes of $\mathrm{Sb}$ is inverted for the isomeric/ground state, which is consistent with the complementary IYR measured in this case.

\section{Conclusion and outlook}

Relative CFY of five Sn isotopes $(A=128-132)$ and relative IFY of six Sb isotopes $(A=128-133)$ were measured in the first systematic high-energy neutron-induced fission yield measurement at the upgraded IGISOL-4 facility, at JYFL.

The white neutron field, extending up to about $30 \mathrm{MeV}$ and with a cross-section weighted average energy of $(12.4 \pm$ 8.8) $\mathrm{MeV}$, impinged on a nat $\mathrm{U}$ target. Fission products were mass-separated using the IGISOL technique and transported to a tape implantation station where the nuclides were identified with $\gamma$-spectroscopy.

CFY of Sn show a staggered behaviour not observed in evaluated data libraries and not reproduced by the simulation code GEF. For the IFY of Sb, we find a distribution peaking at $A=132$. This contradicts the high-energy ENDF/B-VII.1 evaluation for the ${ }^{238} \mathrm{U}(\mathrm{n}, \mathrm{f})$ reaction. Our data are instead in better agreement with the GEF calculation, showing the yield increasing with mass in the range $A=128-133$.

Once the MCC30/15 with its higher primary proton currents will be fully operational, the fission products will be transported to the JYFLTRAP Penning trap; here, the identification of the nuclides will not be conditioned on the suitable half-lives, thus allowing a more comprehensive study and reducing the uncertainty due to incomplete decay data.
This work was supported by the European Commission within the Seventh Framework Programme through Fission-2013CHANDA (project No. 605203), by the Swedish Radiation Safety Authority (SSM), by the Swedish Nuclear Fuel and Waste Management Co. (SKB), and by the Academy of Finland under the Finnish Centre of Excellence Programme 20122017 (Nuclear and Accelerator Based Physics Research at JYFL). AK acknowledges support from the Academy of Finland under project No. 275389.

Open Access This is an open access article distributed under the terms of the Creative Commons Attribution License (http://creativecommons.org/licenses/by/4.0), which permits unrestricted use, distribution, and reproduction in any medium, provided the original work is properly cited.

\section{References}

1. A. Solders et al., Nucl. Data Sheets 119, 338 (2014).

2. P. Dimitriou et al., Fission Yields: Current status $\mathcal{E}$ perspective, Technical Report INDC(NDS)-0713 (IAEA, 2016).

3. I. Moore et al., Nucl. Instrum. Methods Phys. Res. B 317, 208 (2013).

4. J. Wilson et al., Phys. Rev. Lett. 118, 222501 (2017).

5. H. Penttilä et al., Eur. Phys. J. A 52, 104 (2016).

6. D. Gorelov, Nuclear fission studies with the IGISOL method and JYFLTRAP, $\mathrm{PhD}$ Thesis, University of Jyväskylä (2015).

7. A. Mattera et al., Eur. Phys. J. A 53, 173 (2017).

8. D. Gorelov et al., Nucl. Instrum. Methods Phys. Res. B 376, 46 (2016).

9. K.-H. Schmidt et al., Nucl. Data Sheets 131, 107 (2016).

10. A. Mattera, Characterization of a Neutron Source for Fission Yields Studies, Licentiate Thesis, Uppsala (2014).

11. K. Jansson, A. Al-Adili, N. Nilsson, M. Norlin, A. Solders, Eur. Phys. J. A 53, 243 (2017).

12. P. Karvonen et al., Nucl. Instrum. Methods Phys. Res. B 266, 4794 (2008).

13. J. Fitzgerald et al., FitzPeaks gamma analysis and calibration software (2009), http://www.jimfitz.co.uk/ fitzpeak.htm.

14. A. Mattera, nIGISOL-2016. Technical report, Uppsala University (2017). available at: http://urn.kb.se/ resolve?urn=urn:nbn:se:uu:diva-326306.

15. A. Al-Adili et al., Eur. Phys. J. A 51, 59 (2015).

16. National Nuclear Data Center, Chart of Nuclides database, http://www.nndc.bnl.gov/chart/, accessed: 03/2017.

17. Japan Atomic Energy Agency, Nuclear Data Center, Chart of the Nuclides 2014, http://wwwndc.jaea.go.jp/ $\mathrm{NuC} /$ index_J.html, accessed: 03/2017.

18. J. Magill et al., Karlsruher Nuklidkarte (1998).

19. V. Rakopoulos et al., EPJ Web of Conferences 146, 04054 (2017). 